\title{
Calcul direct, sans itération, de la perte de charge en conduite par la formule de Colebrook
}

\section{J. Nackab}

(BP 32, 78670 Villennes-sur-Seine)

La formule empirique de Colebrook, formule universelle de calcul des pertes de charge des conduites industrielles de section circulaire en régime turbulent, s'écrit

$$
\lambda=\left[2 \log _{10}\left(\frac{0,27}{D / k}+\frac{2,51}{\operatorname{Re} \sqrt{\lambda}}\right)\right]^{-2}=f\left(\frac{D}{k}, \operatorname{Re}, \lambda\right)
$$

$\lambda$ est le coefficient de pertes de charge, celle-ci $-\Delta H$ en hauteur du fluide - étant donnée par la relation

$$
\Delta H=\lambda \frac{L}{D} \frac{V^{2}}{2 g}
$$

$L$ est la longueur de la conduite, $D$ son diamètre, $V$ la vitesse moyenne de l'écoulement.

Dans la formule (1), $k$ est l'épaisseur des rugosités de la paroi, et Re le nombre de Reynolds de l'écoulement (Re $=V D / v, v$ étant la viscosité cinématique du fluide).
La formule (1), formule de Colebrook, a l'inconvénient de comporter la grandeur recherchée $\lambda$ dans des deux membres, ce qui conduit à une résolution par approximations successives.

Pour transformer ce calcul par itérations en calcul direct, nous avons essayé de trouver la valeur $\lambda_{0}$ qui, introduite dans le deuxième membre, conduirait à une valeur de $\lambda$ aussi voisine que possible de la valeur donnée par (1). Nous avons pensé prendre pour $\lambda_{0}$ la valeur de $\lambda$ correspondant au régime lisse $(D / k$ très grand). Cette valeur est aussi donnée par une formule implicite, mais l'approximation ci-dessous est excellente

$$
\lambda_{0}=0,4 \operatorname{Re}^{-0,3}+0,0053=f(\operatorname{Re})
$$

La formule (1) devient alors

$$
\lambda=\left[2 \log _{10}\left(\frac{0,27}{D / k}+\frac{2,51}{\operatorname{Re} \sqrt{0,4 \operatorname{Re}^{-0,3}+0,0053}}\right)\right]^{-2}=f\left(\frac{D}{k}, \operatorname{Re}\right)
$$

Dans le tableau ci-dessous, le chiffre supérieur est, en $\%$, le $\lambda$ calculé au moyen de la formule explicite (3), le chiffre inférieur étant calculé à partir de la formule de Colebrook (1) par approximations successives, dans la gamme des $\mathrm{D} / \mathrm{k}$ et Re correspondant aux utilisations habituelles.

On voit que l'emploi de la formule (3) donne des valeurs tout à fait acceptables.

\begin{tabular}{|r|r|r|r|r|r|r|}
\hline \multicolumn{1}{|r|}{ Re } & $10^{3}$ & \multicolumn{1}{|c|}{$10^{4}$} & $10^{5}$ & $10^{6}$ & $10^{7}$ & $10^{8}$ \\
\hline 10 & 12.3 & 10.5 & 10.2 & 10.2 & 10.2 & 10.2 \\
& 11.7 & 10.3 & 10.2 & 10.2 & 10.2 & 10.2 \\
\hline $10^{2}$ & 6.3 & 4.1 & 3.8 & 3.8 & 3.8 & 3.8 \\
& 6.8 & 4.3 & 3.9 & 3.8 & 3.8 & 3.8 \\
\hline $10^{3}$ & 6.2 & 3.1 & 2.2 & 2.0 & 2.0 & 2.0 \\
& 6.3 & 3.2 & 2.2 & 2.0 & 2.0 & 2.0 \\
\hline $10^{4}$ & 6.3 & 3.1 & 1.8 & 1.3 & 1.2 & 1.2 \\
& 6.3 & 3.1 & 1.9 & 1.3 & 1.2 & 1.2 \\
\hline $10^{5}$ & 6.3 & 3.1 & 1.8 & 1.2 & 0.9 & 0.8 \\
& 6.3 & 3.1 & 1.8 & 1.2 & 0.9 & 0.8 \\
\hline $10^{6}$ & 6.3 & 3.1 & 1.8 & 1.2 & 0.8 & 0.6 \\
& 6.3 & 3.1 & 1.8 & 1.2 & 0.8 & 0.6 \\
\hline
\end{tabular}

